\title{
Conservative Treatment of Intraosseous Hemangiomas in the Mandible: Case Report with a 17-Year Follow-up Period
}

\author{
${ }^{1}$ Fausto Frizzera, ${ }^{2}$ Ivette Beccalli, ${ }^{3}$ Rosa Maria Lourenço Carlos Maia, ${ }^{4}$ Mateus Rodrigues Tonetto
}

${ }^{5}$ Liliane Scheidegger da Silva Zanetti, ${ }^{6}$ Liliana Pimenta Aparecida de Barros

\begin{abstract}
Intraosseous hemangiomas in the jaws are rare lesions and may lead to several complications. The authors present a case of a 12-year-old girl with a radiolucent periapical lesion between tooth 35 and 36 where nocturnal exsanguinating bleeding started to occur from periodontal sulcus during orthodontic treatment. Diagnosis of an intraosseous hemangioma in the mandible was based on positive needle aspiration for blood, computed tomography and arteriography. At first the family chose to only follow-up the lesion but episodes of nocturnal hemorrhage were becoming more frequent and a treatment was requested. Embolization and dental extraction were performed in order to treat the lesion. After a follow-up period of 17 years no more cases of hemorrhage occurred and lesion regressed.
\end{abstract}

Keywords: Angiography, Hemangioma, Case report, Embolization, Follow-up studies.

How to cite this article: Frizzera F, Beccalli I, Maia RMLC, Tonetto MR, da Silva Zanetti LS, de Barros LPA. Conservative Treatment of Intraosseous Hemangiomas in the Mandible: Case Report with a 17-Year Follow-up Period. J Contemp Dent Pract 2014;15(3):376-380.

\section{Source of support: Nil}

Conflict of interest: None declared

\section{INTRODUCTION}

Vascular tumors are originated from endothelial cell proliferation and include hemangioma (HEM) of the childhood, rapidly involuting congenital hemangioma, noninvoluting congenital hemangioma, tufted angioma, kaposiform hemangioendothelioma and pyogenic granuloma. Commonly located in cutaneous soft tissues, HEMs have a proliferative

\footnotetext{
${ }^{1,5}$ Full Professor, ${ }^{2,3,6}$ Adjunct Professor, ${ }^{4}$ Professor

${ }^{1,5}$ Department of Dentistry, Integrated College Espírito Santense FAESA, Vitória, ES, Brazil

${ }^{2,3,6}$ Department of Clinical Odontology, Health Sciences Center, Federal University of Espírito Santo, Vitória, ES, Brazil

${ }^{4}$ Postgraduate Course in Dentistry, University of Cuiabá Cuiabá, MT, Brazil

Corresponding Author: Liliana Pimenta Aparecida de Barros Adjunct Professor, Department of Clinical Odontology, Health Sciences Center, Federal University of Espírito Santo, Vitória ES, Brazil, Phone: (27) 33357234, e-mail: lilianabarros@ hotmail.com
}

phase, followed by slow spontaneous involution, which can take years. In some cases HEM may lead to compression of surrounding structures, pain, formation of fissures, ulcers or hemorrhages and it can cause functional and esthetic problems, requiring specific treatment. ${ }^{5}$

Primary vascular tumors of bone are rare. Hemangiomas are uncommon intraosseous lesions. They appear as incidental findings in the skull or in the spine, incidence ranges from 0.5 to $1 \%$ of all osseous neoplasms. ${ }^{1}$ Primary mandibular lesions have been rarely reported in infancy and early childhood, showing peak incidence at puberty, no spontaneous regression has been described. ${ }^{4}$

Among the facial bones, the mandible is more affected than the maxilla and the nasal bones. ${ }^{9}$ The sites of predilection are the molar and premolar region accounting for $65 \%$ of cases and there is a high predominance in females. ${ }^{3}$ Hemangiomas have an excellent prognosis and have a low rate of local recurrence. Maxillary HEMs are frequently located close to the teeth, even a simple dental procedure in this cases, like the extraction of mobile teeth, has a risk of generating an uncontrollable bleeding, that might even lead to death. ${ }^{10}$

The authors report a case of a patient with nocturnal hemorrhage due to an intraosseous HEM in the mandible. Treatment was performed in a conservative manner and a follow-up period of 17 years is presented where no more bleeding episodes occurred.

\section{CASE REPORT}

A 12-year-old girl presented mobility in the tooth 35 during orthodontic treatment. There was no clinical sign or symptom (Fig. 1A), periapical radiography showed late root development of the tooth 35 and apical radiolucency between teeth 35 and 36 (Fig. 1B). In order to have a conclusive diagnosis an incisional biopsy was going to be performed, but during the procedure there was positive needle aspiration for blood and intensive hemorrhage occurred, it was identified the presence of an intraosseous HEM and the procedure was aborted. The hemorrhage was controlled using compression and occlusive suture. More radiographic exams were requested (Figs 1C and D).

Computed tomography scan showed a lytic lesion in the left mandibular body and areas with homogeneity and 

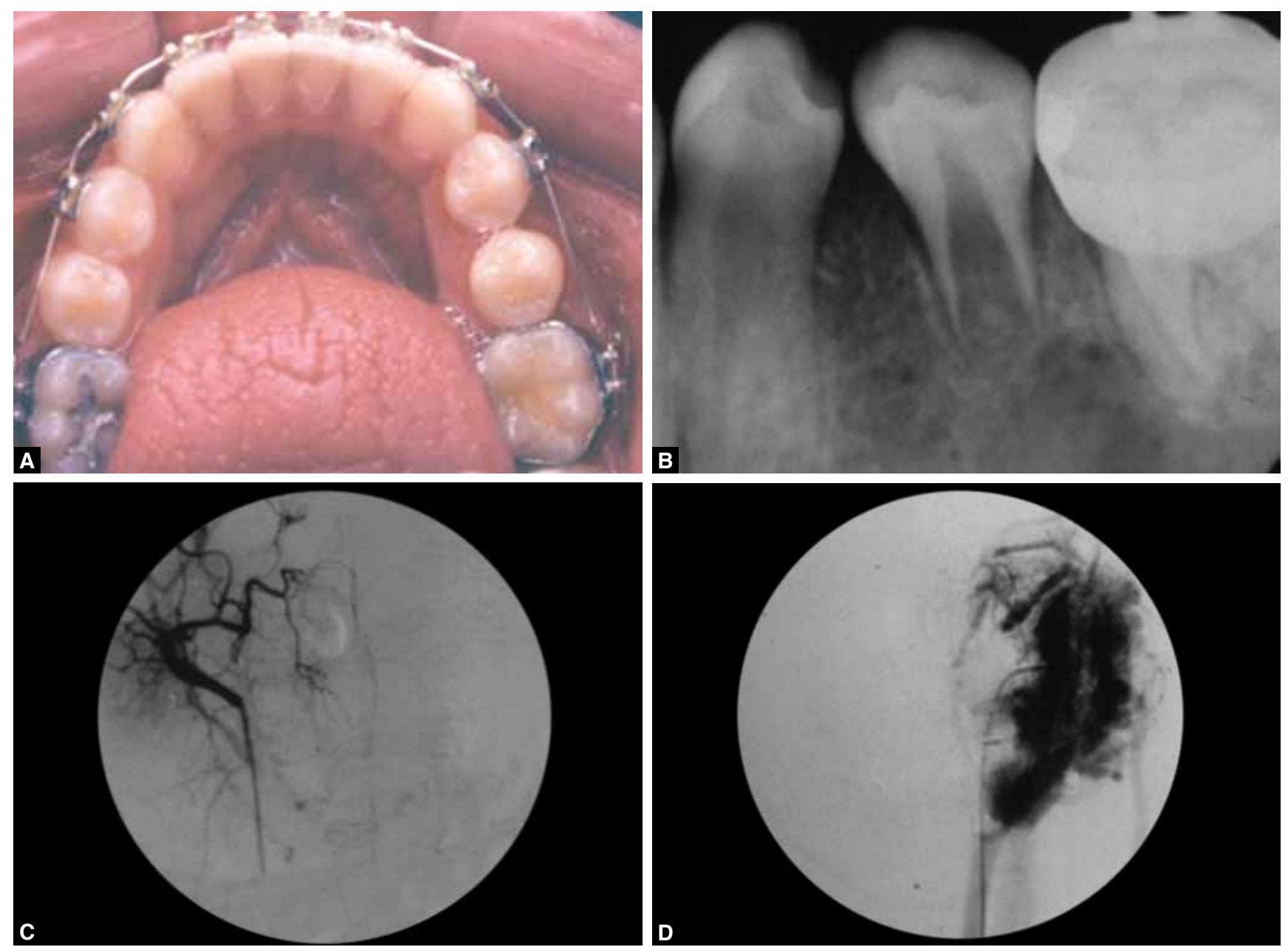

Figs 1A to D: (A) No major alterations could be detected on clinical examination, (B) late root development of tooth 35 and radiolucent lesion between teeth 35 and 36, (C) angiography of right external carotid presenting normal appearance, (D) angiography of left external carotid showing the presence of an intraosseous HEM with high-flow, high-shunt, in the left mandibular ramus

density similar to soft tissue in your interior. It also presented a slight increase of the soft tissue close to the lesion. Vascular anomalies could not be identified. Cintilography demonstrated an abnormal hypercaptation of the isotope in the left hemimandible and an increased vascularization of the lesion.

Patient's family opted to follow-up the lesion instead of doing any kind of procedure. After 6 months, cases of nocturnal hemorrhage from the periodontal sulcus started to happen. These episodes were becoming frequent and the family requested a treatment and embolization of the lesion was suggested.

A new head CT scan and angiography were made (Figs $2 \mathrm{~A}$ and $\mathrm{C})$. In the first exam it was seen the presence of an extensive osteolytic lesion in the left hemimandible, involving body and ramus, with lobulation in your interior. This bone was expanded and the lesion was inside the diploe, but the cortical was thin and with discontinuity in some areas. The interior of the lesion also presented soft tissue density. The facial angiography showed an intraosseous HEM with principal vascularization from the inferior alveolar artery. Initial portion of the lesion presented a direct arteriovenous communication and a large vein ectasy in bone interior. Inferior alveolar vein showed signs of thrombosis in the distal portion where it connects with the intern maxillary vein, causing the opacification of several others veins that were draining the lesion. Arteries originated from the left facial and lingual artery showed direct fistulas. Lesion high flow produced 'stealing' phenomenum. Normal soft tissues of the hemiface were irrigated only by collateral irrigation from contralateral arteries.

Embolization consisted of four direct punctions, after each one it was performed an angiographic study in the left external carotid and after fourth punction in the right lingual artery. The procedure was satisfactory after four direct punctions in the drainage system of the HEM. To complement the embolization particles of polyvinyl alcohol foam (IVALON) of 600 and 1000 micra were used in the left lingual artery and in the external carotid artery (Fig. 2C). An angiographic study was performed 6 days later and showed a superficial shunt with low-flow lesion, with opacification in the left jugal region, but no sign of opacification of the intraosseous HEM was seen. 


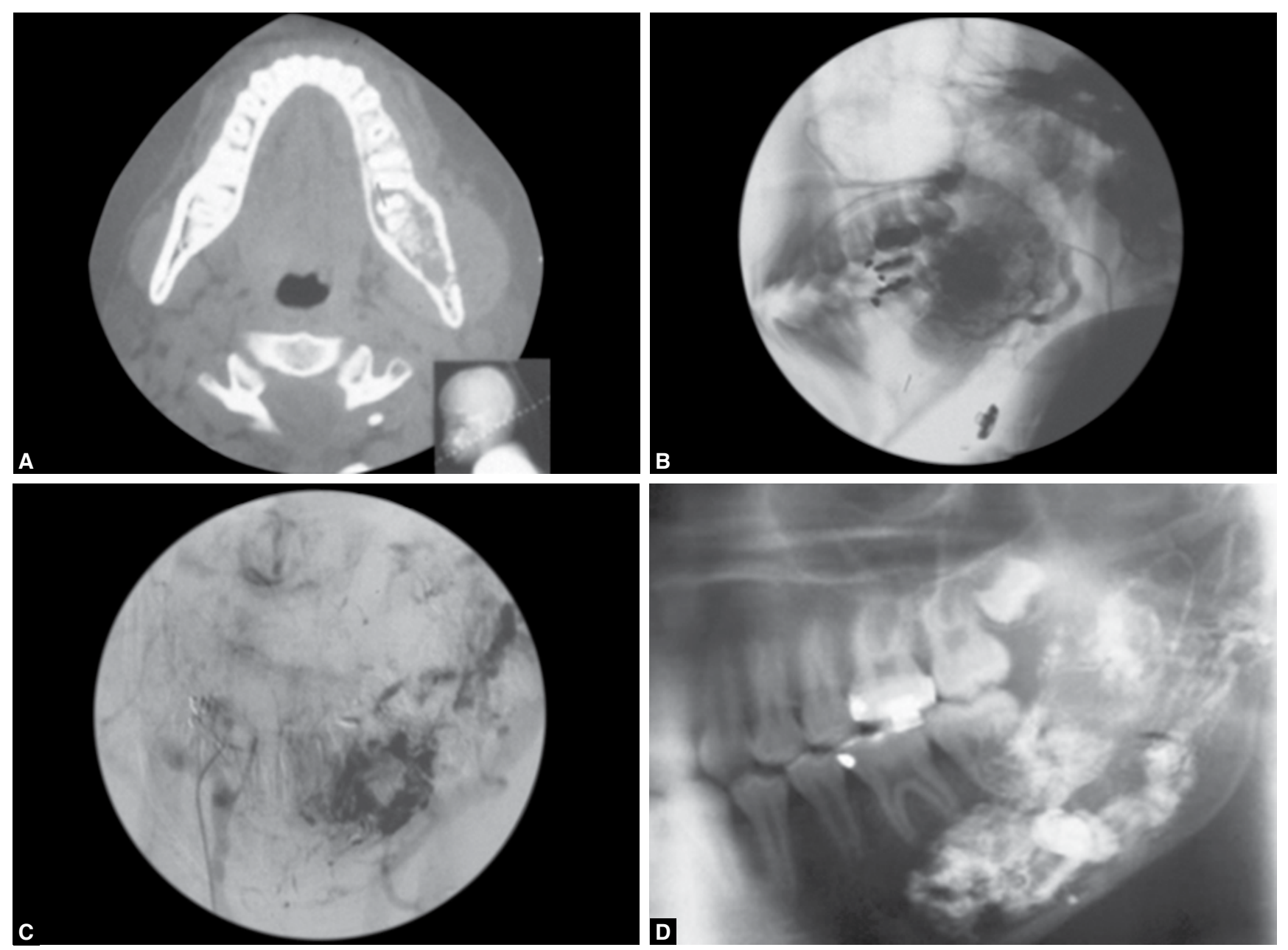

Figs 2A to D: (A) Mandible CT scan showing a slight increase of the left mandible and a lytic lesion in left mandibular body, (B) angiographic study in a lateral view of left external carotid showing the presence of the lesion and communications, (C) aspect of left carotid angiography after the fourth punction and (D) panoramic radiograph after first embolization

The child remained well for the next 3 months, but episodes of bleeding started to happen again. Two different approaches were suggested to the family: resection of left hemimandible or embolization associated with extraction of left mandibular molars $(36,37)$. The family preferred the more conservative procedure and another embolization was done associated with teeth extraction (Fig. 3A).

Six months later another facial angiography showed complete occlusion of the lesion (Fig. 4A). It only showed few niduses in soft tissues of the left jugal region and intense capillary opacification, clinically it was seen hyperemy in the region.

After second embolization and extraction of left mandibular molars the patient and his parents did not report any other case of hemorrhage and were completely satisfied with the results. These favorable signs convinced us to follow her up without any other intervention (Figs 3B to D). Seventeen years have passed, new exams were performed (Figs 4B to D) and there has not been any report of bleeding, representing the success of the treatment.

\section{DISCUSSION}

HEM of bone may be life-threatening if improperly managed. A simple dental procedure, like extraction of mobile tooth, or even the eruption of succedaneous permanent tooth can produce uncontrollable bleeding, that may even lead to death. ${ }^{4}$

Clinical diagnosis is often inaccurate. Some of the signs and symptoms of this vascular lesion are: expansion of bone, facial asymmetry, swelling, pain, earache or pain in the TMJ, paresthesia, bruit or pulsation, gingival bleeding, loose teeth and migration, bone resorption, root resorption and the teeth may exhibit a 'pumping action', when depressed in an apical direction it rapidly resume their original position. ${ }^{1,3,4,8}$ It also may not present any signs or symptoms. ${ }^{1,3}$ Hypermobility of the teeth with spontaneous hemorrhage from the gingival sulcus is the most significant morbidity for patients, especially in mixed dentition period. ${ }^{1,3,4,8}$ Modern radiographic techniques and image diagnosis, such as CT scan and MRI images, play a decisive role in diagnosis. When intraosseous HEM is suspected the association of exact history-taking, physical examination and radiographic studies, such as caro- 

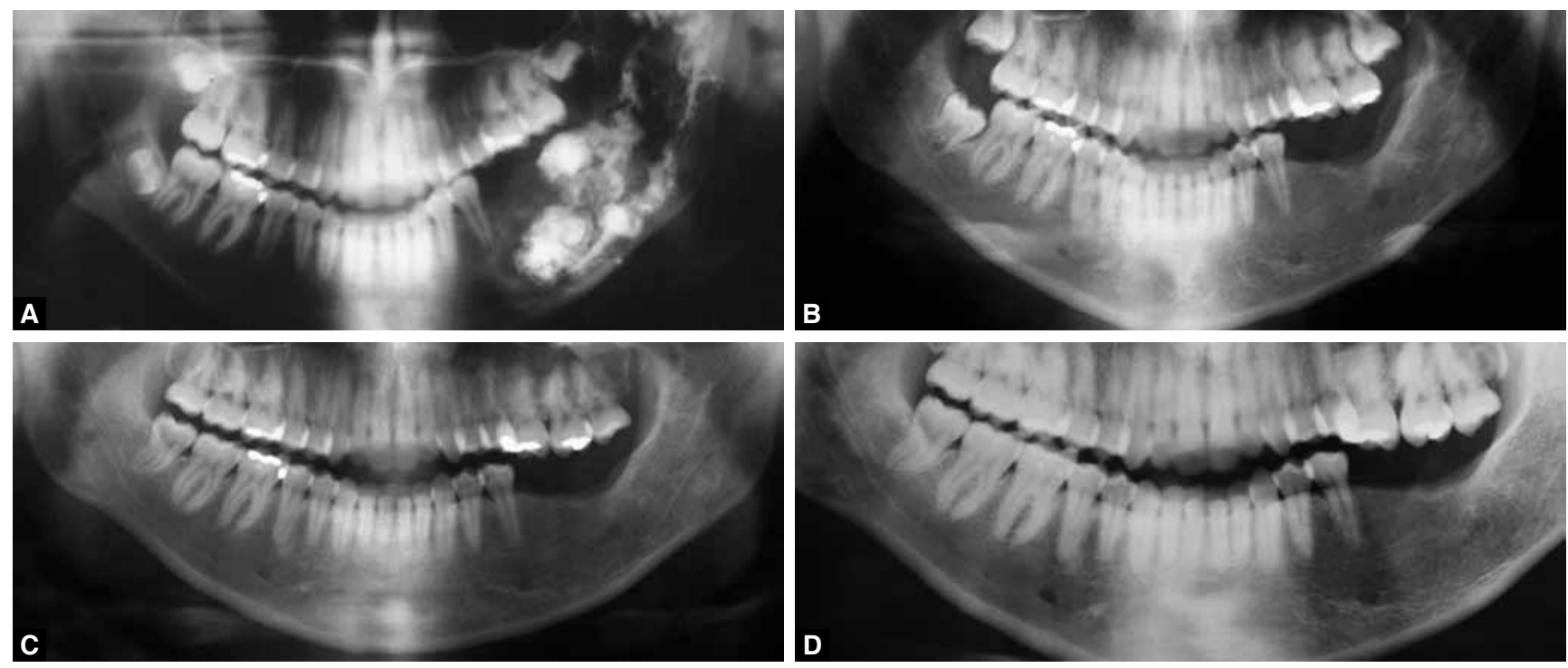

Figs 3A to D: (A) Panoramic radiograph after the second embolization and extraction of the two left mandibular molars, (B) control panoramic radiograph, 5 years after the second embolization, $(C)$ control panoramic radiograph, 10 years after treatment and (D) control panoramic radiograph, 16 years after treatment
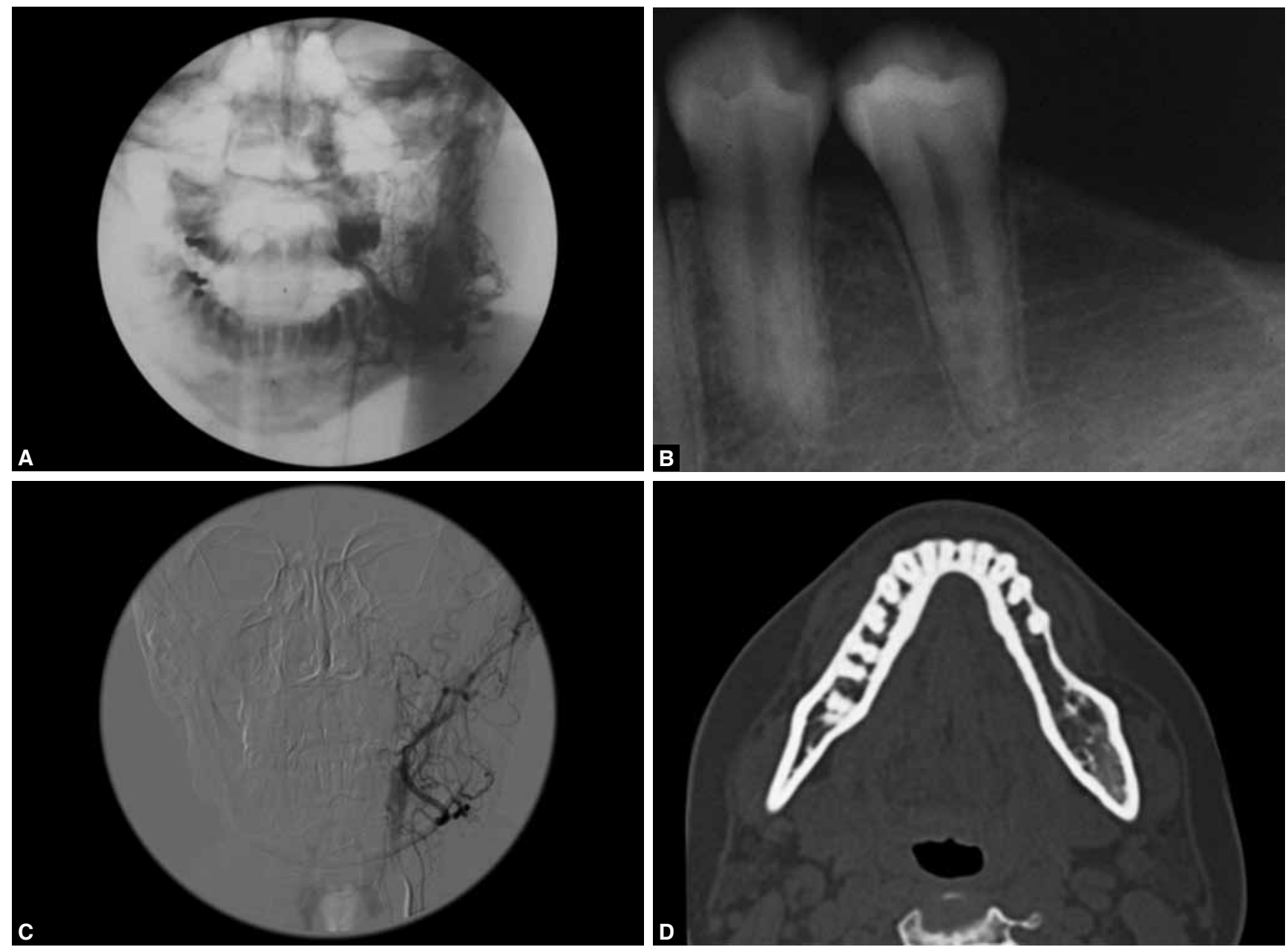

Figs 4A to D: (A) Angiography of external left carotid, 6 months after second embolization, (B) periapical radiograph showing normal bone aspect after 17 years, clinically there was no mobility, $(C)$ arteriography performed 17 years after second embolization showing stability of the results and (D) CT scan 17 years after treatment

tid angiography, clarifies the diagnosis in more than $90 \%$ of the cases. ${ }^{10}$ In the present case mobility of the tooth 35 and its late root development was only seen during orthodontic treatment and no other sign or symptom was present. The presence of an intraosseous HEM was only identified during the attempt to perform an incisional biopsy. 
Biopsy, arterial ligature and radiotherapy should be avoided when dealing with intraosseous HEM. ${ }^{4}$ Ligation of the external carotid can produce lesion enlargement. ${ }^{1} \mathrm{~A}$ study of 12.000 children found that irradiation of a vascular lesion of the skin increased the risk of central nervous system or thyroid cancer significantly. ${ }^{4} \mathrm{An}$ incisional or excisional biopsy results in a definitive diagnosis but should be avoided when a vascular lesion is suspected. This procedure can cause sudden loss of large quantities of blood. ${ }^{1,4,7}$ Needle aspiration can help with the diagnosis, positive aspiration for blood is a first step in diagnosis. In our case an incisional biopsy was going to be performed but intense exsanguination occurred after positive needle aspiration, the presence of a vascular lesion was identified and procedure was aborted. Appropriate radiographic exams were requested to clarify diagnosis and continue the treatment.

Intraosseous HEM present a wide variety of radiographic appearances, such as a unilocular lytic lesion, a well defined cystic space with a sclerotic rim, a bony trabeculae radiating from the center of the lesion and a honeycombed appearance. ${ }^{3,8}$ Cortical involvement can be visualized clearly with CT scan. It also shows thinning or expansion of cortices and the radiodensity of the contents of the lesion. ${ }^{3}$ Differential diagnosis of multilocular HEM of bone includes ameloblastoma, odontogenic myxoma, odontogenic keratocyst, central giant cell granuloma and aneurysmal bone cyst. The unilocular lesion can be confused with other cystic processes. ${ }^{1}$

Angiography is a useful adjunct to diagnosis when clinical and radiologic findings suggest possibility of a HEM in the jaws bone. This will delineate the boundaries and arterial connections of the lesion. ${ }^{1}$ Preoperative embolization and intervention by different therapeutic surgical modalities became treatment of choice in majority of cases. Thrombosis of vascular tumors and malformations can be promoted by embolization, which minimizes bleeding, decreases the need of intraoperative transfusions and facilitates surgical approaches if necessary. ${ }^{6}$

During a long period, surgery was the only treatment of choice of intraosseous HEM, severe hemorrhage has been described when this procedure was carried out alone. ${ }^{2}$ Curettage, embolization, injection of sclerosing solutions and resection have been reported to be successful. To choose the right treatment the vascular supply its size and location must be evaluated. Some authors ${ }^{4}$ studied the modification of the treatment of choice along the years. There were three distinct treatment plans which depended on when the patient was treated, before 1970 it was exclusively chosen surgical treatment with resection, between 1979 and 1983 the resection was performed associated with interventional radiology and between the years of 1987 and 1996 interventional radiology was chosen as an exclusively treatment. Arterial embolization and transcutaneous transosseous embolization permitted different approaches. ${ }^{4}$
The major method suggested by literature has been embolization alone or combined with surgery. Radical resection causes esthetic problems and dysfunction of the mastification system. ${ }^{1,6}$ Treating vascular tumors and malformations by selective embolization has provided in some cases, complete regression of the lesion and made surgery unnecessary. It is imperative to preserve function and esthetics, especially in juvenile patients. Success was reported in a conservative treatment with nidus direct punctures and embolization with embolic material, n-butyl-cyanoacrylate (NBCA) associated with the extraction of mandibular molars, with a follow-up of 3 years. ${ }^{6}$ The present case shows a conservative treatment with direct puncture of the nidus, associated with embolization with particles of IVALON of 600 and 1000 micra and left mandibular molars extraction with a follow-up of 17 years.

\section{CONCLUSION}

It is important to establish that each patient requires individual planning and treatment through a conservative approach should always be considered. Both radiologist and surgeon should be aware of indications and limitations of the techniques. Improved knowledge of the development of these lesions and collaboration between radiologists and surgeons improves treatment outcomes for intraosseous HEM lesions.

\section{REFERENCES}

1. Beziat JL, Marcelino JP, Bascoulergue Y, Vitrey D. Central vascular malformation of the mandible: a case report. J Oral Maxillofac Surg 1997;55:415-419.

2. Cheng CK, Gaia BF, de Oliveira Neto HG, Martini MZ, Aburad A, Shinohara EH. Severe hemorrhage during an incisional biopsy: a report of a case. J Contempo Dent Pract 2007;8(3):97-103.

3. Fernandéz LR, Luberti RF, Domínguez FV. Aspectos radiográficos de los hemangiomas óseos maxilofaciales. Revisión bibliográfica y presentación de dos Casos. Med Oral 2003;8:166-177.

4. Giaoui, et al. Treatment of vascular malformations of the mandible. Int J Oral Maxillofac Surg 2003;32:132-136.

5. Hayashi N, Masumoto T, Okubo T. Hemangiomas in the face and extremities: MR-guided sclerotherapy-optimization with monitoring of signal intensity changes in vivo. Radiology 2003; 226(2):567-572.

6. Kaneko R, Tohnai I, Ueda M, Negoro M, Yoshida J, Yamada Y. Curative treatment of central hemangioma in the mandible by direct puncture and embolization with n-butyl-cyanoacrylate (NBCA). Oral Oncol 2001;37(7):605-608.

7. Kim HJ, Kim JH, Kim JH, Hwang EG. Bone erosion caused by sinonasal cavernous hemangioma: CT findings in two patients. AJNR 1995;16:1176-1178.

8. Mohammadi H, Said-Al-Naief NA, Heffez LB, Arteriovenous malformation of the mandible. Oral Surg Oral Med Oral Pathol Oral Radiol Endod 1997;84:286-289.

9. Moore SL, Chun JK, Mitre SA, Som PM. Intraosseous hemangioma of the zygoma: CT and MR Findings, AJNR Am J Neuroradiol 2001;22(7)1383-1385.

10. Williams HJ, Wake MJ, John PR. Intraosseous haemangioma of the mandible: a case report. Pediatr Radiol 2002;32:605-608. 